\title{
Brillouin scattering near the ferroelectric phase transition in TSCC
}

\section{Authors: T. Hikita, P. Schnackenberg, \& V. Hugo Schmidt}

This is an Accepted Manuscript of an article published in Ferroelectrics in 1985, available online: http://www.tandfonline.com/10.1080/00150198508221390.

T. Hikita, P. Schnackenberg, and V.H. Schmidt, "Brillouin scattering near the ferroelectric phase transition in TSCC," Ferroelectrics 63, 107-114 (1985).

http://dx.doi.org/10.1080/00150198508221390

Made available through Montana State University's ScholarWorks

scholarworks. montana.edu 


\title{
BRILLOUIN SCATTERING NEAR THE FERROELECTRIC PHASE TRANSITION IN TSCC
}

TOMOYUKI HIKITA*, PAUL SCHNACKENBERG AND V. HUGO SCHMIDT Physics Department, Montana State University, Bozeman, Montana 59717 U.S.A.

\begin{abstract}
Brillouin spectra from longttudinal phonons in ferroelectric tris-sarcosine calcium chloride (TSCC) propagating along $[100],[010]$ and $[001]$ have been measured as functions of temperature. Large anomalies were found in the Brillouin shift and linewidth in the $[100]$ and $[001]$ phonons. These anomalies are interpreted as arising from the linear coupling of the polarization and phonons. From the the temperature where the linewidth is maximum, the relaxation time of the polarization fluctuations is estimated to be $T=3.1 \times 10^{-12} /\left(\mathrm{T}_{\mathrm{c}}-\mathrm{T}\right) \mathrm{sec}$, where $\mathrm{T}_{c}$ is the ferroelectric transition temperature. We also observed anomalies in Brillouin shift and linewidth of the [010] phonons which propagate along the ferroelectric axis. These anomalies are interpreted as roming from electrostrictive coupling. The energy relaxatior time wis estimated to be $\tau_{E}=2.5$ $x 10^{-1} /\left(T-T_{c}\right)$ sec in the pardelectric (PE) phase and $\tau_{E}=1.0$ $x 10^{-9} /\left(T-T_{c}\right)$ sec in the ferroelectric (FE) phase, by comparing our Brillouin results with those $f$ the ultrasonic measurements.
\end{abstract}

\section{INTRODUCTION}

Tris-sarcosine calcium chloride (TSCC) is a uniaxial ferroelectric, with a Curie temperature near $130 \mathrm{~K} .^{1}$ At room temperature it has orthorhombic symmetry characterized by space group $\mathrm{D}_{2} \mathrm{~h}^{16}$-Pnma with four formula units per unit cell. ${ }^{2}$ In the FE phase this crystal remains orthorhombic with spase group $\mathrm{C}_{2}{ }^{9}-\mathrm{Pna}_{1}$.

There are disputes concerning whether the FE transition of this crystal is order-disorder or displacive. Soft modes have been found in TSCC both in the PE and FE phases. ${ }^{3-5}$ Scott's group claims that this crystal is a textbook example of a displacive type ferroelectric from their analysis of the results of millimeter wavelength spec- 
troscopy. ${ }^{5}$ They could satisfactorily explain the temperature dependence of the dielectric constant in the above frequency region by a simple oscillator model. However, Chen and Schaack recently showed that their Raman and infrared spectra can be much better explained by the pseudospin-phonon coupling tkeory. ${ }^{6,7}$ They concluded that TSCC and partially brominated TSCC (i.e. $\operatorname{TSCC}_{1-x_{x}} B_{x}$ undergo phase transitions which are mostly of the order-disorder type. 6,7 Furthermore, Schmidt showed that the pressure-temperature phase diagram $^{8}$ of TSCC can be interpreted by a cluster model, ${ }^{9}$ which indicates that this phase transition is partly of the order-disorder type. Since Brillouin scattering measures the velocity and attenuation of acoustic phonons in the $\mathrm{GHz}$ region, its use in TSCC helps to understand the dynamical behavior in this frequency region.

\section{EXPERIMENTAL}

Single crystals of TSCC were grown from an aqueous solution of pure sarcsine and reagent grade $\mathrm{CaCl}_{2}$. Since this crystal has ferroelastic domains at room temperature, single domain portions were carefully examined with a polarizing microscope. Exceptionally clear parts were chosen from these crystals and two samples which have the form of a rectangular parallelepiped $3 \times 3 \times 2 \mathrm{~mm}^{3}$ were prepared. One sample was cut so that the longitudinal phonons $q \|[100]$ and $q \|[010]$ could be measured by right angle scattering and the other was cut so that longitudinal phonons $q \|[001]$ and $q \|[010]$ could be measured. The two samples were polished and then masked together by a blackened coper foil with pinholes for the incident and the scattered 1 ight beams. The masked sample pair was placed in a high pressure optical cell described previously. ${ }^{10}$ The cell was filled with isopentane for index matching. The optical cell temperature could be controlled within $3 \mathrm{mK}$ during the measurement.

A Lexel Model 95-2 argon laser operating in a single mode at a wavelength of $514.5 \mathrm{~nm}$ and a power level of 200 to $300 \mathrm{~mW}$ was used as a light source. The scattered light was collected in a cone of 
$1^{\circ}$ and analyzed by a piezoelectrically scanned Burleigh Model 140 Fabry-Perot interferometer. Finesse optimization and drift control were achieved by a home-made control system using an AIM-65 microcomputer.

\section{RESULTS AND DISCUSSION}

Figures 1-3 show the temperature dependence of the Brllouin shift and linewidth for longitudinal phonons propagating along [100], [010] and [001]. The temperature dependence of the Brillouin shift and the decay rate of the $[100]$ phonons is quite similar to that of the [001] phonons though the anomlies are smaller for the latter case. In the above two cases, the polarization and the strain couple bilinearly in the FE phase. ${ }^{11}$ For these two phonon propagations, the velocity $v$ and the attenuation in the $F E$ phase are given by ${ }^{12-14}$

$$
v^{2}=v_{\infty}^{2}-\left[\left(v_{\infty}^{2}-v_{0}^{2}\right) /\left(1+\omega^{2} \tau_{0}^{2} t^{-2}\right)\right],
$$

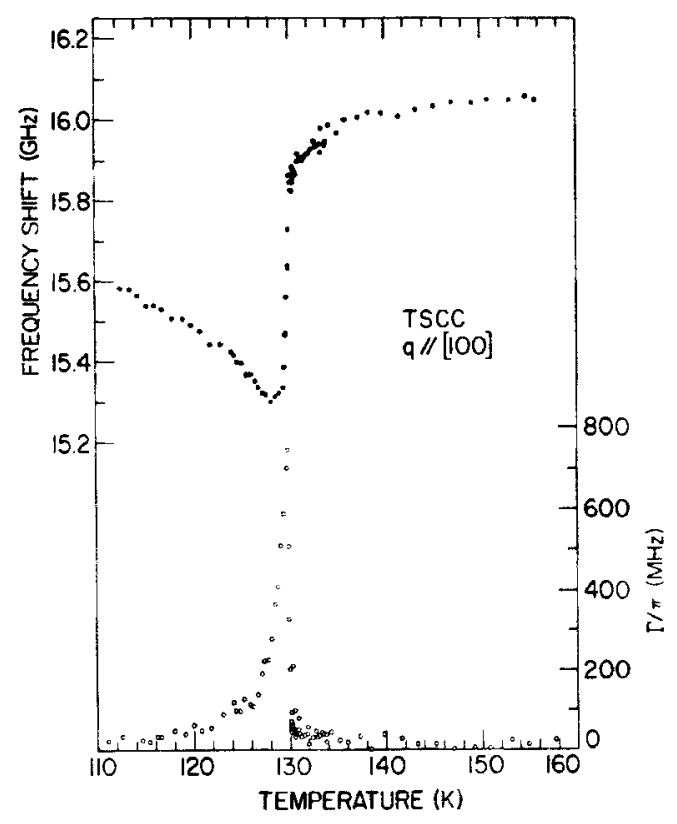

Figure 1 Temperature dependence of Brillouin shift $(\bullet)$ and linewidth (O) (FWHM) of longitudinal phonons in TSCC propagating along the $[100]$ direction. The scattering geometry is $x+y(z z)-x+y$. 


$$
\alpha=\left[\left(v_{\infty}^{2}-v_{0}^{2}\right) / 2 v_{\infty}^{3}\right]\left[\omega^{2} \tau_{0} t^{-1} /\left(1+\omega^{2} \tau_{0}^{2} t^{-2}\right)\right] .
$$

where $t$ is the reduced temperature $\left(T_{c}-T\right) / T_{c}, \tau_{0}$ is the individual dipole relaxation time in the expression $\tau=\tau_{0} t^{-1}$ for the relaxaion time $\tau$. From Eq. (2) we notice that the phase transition is marked by a quick drop in the velocity with decreasing temperature. However, experimentally the $[100]$ and $[001]$ phonon velocities do not exhibit

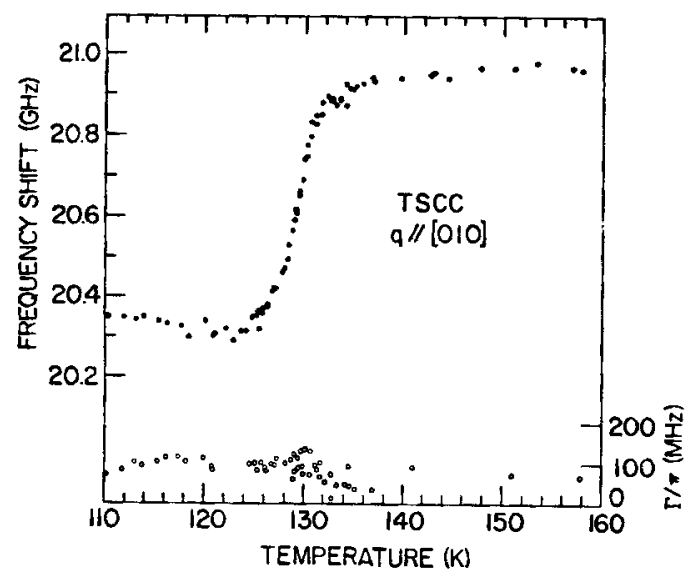

Figure 2 Temperature dependence of Brillouin shift ( $\bullet$ ) and linewidth ( $O$ ) (FWHM) of longitudinal phonons in TSCC propagating along the [010] direction. The scattering geometry is $x+y(z z) x-y$.

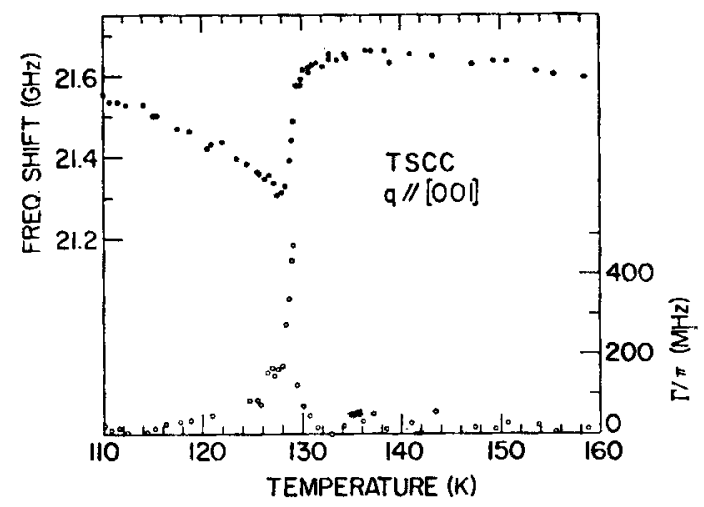

Figure 3 Temperature dependence of Brillouin shift ( $(\bullet)$ and linewidth (O) (FWHM) of longitudinal phonons in TSCC propagating along the [001] direction. The scattering geometry is $x+y(x x) y-z$. 
such sharp drops at $T_{c}$, but rather slow rounding. These anomlies are attributed to the quadratic coupling of the polarization and the strain or in other words, electrostrictive coupling. ${ }^{11,15}$ Such contributions to the anomalies of the velocity and the attenuation are schematically illustrated in Fig. 4. As we see from Fig.4, the temperature $T_{m}$ at which the attenuation coefficient is maximum does not equal $T_{C}$, but occurs somewhat below $T_{c}$. From Eqs. (2) and (3) we obtain the relation $\left(T_{c}-T_{m}\right) / T_{c}=\omega \tau_{0}$. By this relation we can calculate the elementary relaxation time. Though the clear indication of $T_{c}$ is blurred by the additional small anomaly from the electrostrictive contributions, we can use the steepest dropping point of the velocity as $T_{c}$. From the velocity and attenuation for the above two cases, we obtain the following results: For the qll[100] phonons, $\tau_{0}=$

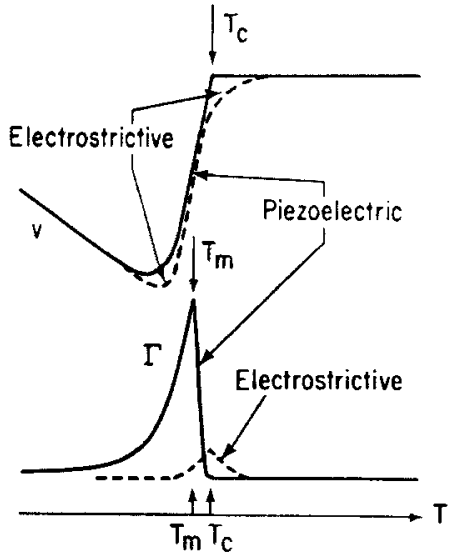

Figure 4 Anomalies in the velocity and the attenuation caused by piezoelectric coupling and electrostrictive coupling for longitudinal phonons propergating perpendicular to the polarization axis.
$2.4 \times 10^{-14} \mathrm{sec}$, and for the q II [001] phonons, $\tau_{0}=2.3 \times 10^{-14} \mathrm{sec}$. These two results agrees well. we will use their average, $\tau_{0}=2.4 \mathrm{x}$ $10^{-14} \mathrm{sec}$.

We next consider the temperature dependence of the Brillouin shift and the phonon decay rate for the longitudinal phonons parallel to the spontaneous polarizaion. In this case, polarization fluctuations do not couple bilinearly to the strain, because of the appearance of the depolarization field in the longitudinal polarizaion wave. ${ }^{15,16}$ Therefore, the anomalies in the velocity and attenuation of these phonons should be expained by quadratic coupling of the polarization to the strain in both $P E$ and $F E$ 
phases.

We now compare the velocity and attenuation of the acoustic waves in the $\mathrm{GHz}$ and $\mathrm{MHz}$ regions. Ultrasonic velocity and attenuation measurements have been performed on this crystal by Sorge and Straube. ${ }^{17}$ Their results are reproduced in Figs. 5 and 6 , toget her with our Brillouin results which are converted to the velocity and attenuation scale.

We use the velocities obtained by Brillouin scattering and ultrasonic measurements for $v_{\infty}$ and $v_{0}$, respectively. We assume the simplest form for the relaxation time to be $\tau_{E}=\tau_{0}^{E} t^{-1}$, where $\tau_{0}^{E}$ is the elementary relaxation time. The $E$ designates that these relaxation times are related to energy rather than polarization fluctuations. By putting into $\mathrm{Eq}$. (3) the values of the attenuation coefficient of the ultrasonic measurements at the temperature $T-T_{c}=$ $=0.9 \mathrm{~K}$ and $-1.3 \mathrm{~K}(2.7 \mathrm{db} / \mathrm{cm}$ and $11.1 \mathrm{db} / \mathrm{cm}$, respectively), we obtain the following values of the relaxation time: $\tau_{0}^{\mathrm{E}}=1.9 \times 10^{-12}$ sec for $T>T_{C}$ and $\tau_{0}^{E}=7.7 \times 10^{-12} \mathrm{sec}$ for $T<T_{C}$.

Chen and Schaack ${ }^{6,7}$ obtaied $\tau_{o}=2.7 \times 10^{-13}$ sec from their analysis of the pseudospin-phonon coupling of Raman and infrared spectra. This value is one order of magnitude larger than ours. Deguchi et a1. ${ }^{18}$ showed that the relaxation time in TSCC is expressed by $\tau=3.8$ $\times 10^{-13}\left(\epsilon_{s}-\epsilon_{\infty}\right)$ sec from their dielectric dispersion measurements. If we use the values $c^{+}=30$ to $50 \mathrm{~K}$ for the Curie constant ${ }^{19,20}$ and the relation $C^{+} / C^{-}=2$, we obtain the values $\tau_{0}=4.4$ to $8.5 \times 10^{-14} \mathrm{sec}$ for the elementary relaxation time which is in relatively good agreement with our values. Though we can obtain an order-parameter relaxation time from the Brillouin scattering experiment, this does not necessarily mean that TSCC undergoes an order-disorder phase transitions. It is also possible to adopt the interpretation that soft mode frequency $\omega_{0}$ decreases and the damping constant $\gamma$ diverges as $\mathrm{T}_{C}$ is approached. In such an extremely damped case, the system has the same correlation function as that of the relaxational mode where the effective relaxation time is given by $\gamma / \omega_{0}^{2}$. Recently, sugo et a1. 21 performed an precise Raman measurements in the ferroelectric 


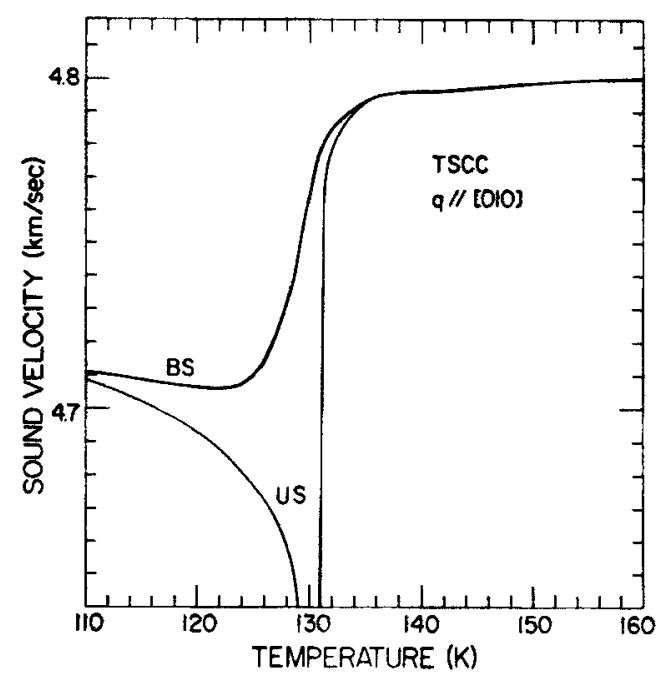

Figure 5 Temperature dependence of sound velocity in TSCC for Brillouin (BS) at $16 \mathrm{GHz}$ and for ultrasonic (US) measurements at $20 \mathrm{MHz}$. The velocity scale for the Brillouin scattering was adjusted to that of the ultrasonic measurements.

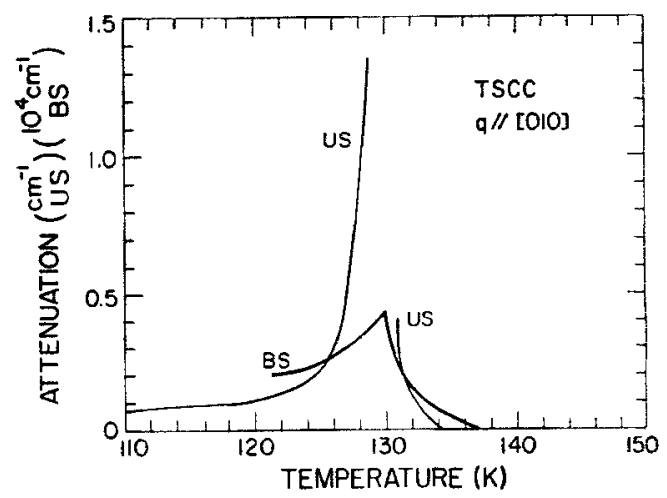

Figure 6 Temperature dependence of the attenuation in TSCC for Brillouin scattering (BS) at $16 \mathrm{GHz}$ and for ultrasonic (US) measurements at $20 \mathrm{MHz}$. 
phase of TSCC up to $T_{c^{*}}$ They showed that $\omega_{0}{ }^{2 / \gamma}$ is nearly proportional to $T_{c}-T$ near $T_{c}$ (within about $10 \mathrm{~K}$ ) and $\tau=5.9 \times 10^{-11} \mathrm{sec}$ at $\mathrm{T}_{c}-0.05 \mathrm{~K}$. Our result gives $\tau=6.3 \times 10^{-11} \mathrm{sec}$ at the same temperature.

\section{REFERENCES}

1. Y. Makita, J. Phys. Soc. Jpn., 20, 2073 (1965).

2. T. Ashida, S. Bando and M. Rakudo, Acta Cryst., B 28, 1560 (1972).

3. S. D. Prokhorova, G. A. Smolensky, I. G. Siny, E. G. Kuzminov,

V. D. Mikvabis and H. Anndt, Ferroelectrics, 25, 629 (1980).

4. G. E. Feldkamp, J. F. Scott and W. Windsch, Ferroelectrics, 39, 1163 (1981).

5. G. V. Kozlov, A. A. Volkov, J. F. Scott, G. E. Feldkamp and

J. Petzelt, Phys, Rev, B, 28, 255 (1983).

6. T. Chen and G. Schaack, to appear in J. Phys, C, 17, (1984).

7. T. Chen and G. Schaack, to appear in J. Phys, $C, 17$, (1984).

8. V. H. Schmidt, Solid State Commun., 35, 649 (1980).

9. V. H. Schmidt, Ferroelectrics, 39, 1151 (1981).

10. M. Yamada and V. H. Schmidt, Rev. Sci. Instr., 49, 1226 (1978).

11. W. Rehwald, Adv. Phys, , 22, 721 (1973).

12. R. W. Gammon and H. 2. Cummins, Phys. Rev. Lett., 17, 193 (1966).

13. T. Yagi, M. Tokunaga and I. Tatsuzaki, J. Phys. Soc. Jpn., 40, 1659 (1976).

14. K. F. Herzfeld and T. A. Litovitz, Absorption and Dispersion of U1trasonic Waves (Academic Press, New York, 1959).

15. K. A. Minaeva, B. A. Strukov and K. Varnstorff, Sov. Phys. Sol. State, 10, 1665 (1969).

16. M. A. Krivoglaz, Sov. Phys, Sol. State, 5, 2526 (1963).

17. G. Sorge and U. Straube, Ferroelectrics, 21, 533 (1978).

18. K. Deguchi, N. Aramaki, E. Nakamura and K. Tanaka, J. Phys. Soc. Jpn., 52, 1897 (1983).

19. E. Sandvold and E. Courtens, Phys. Rev. B, 27, 5660 (1983).

20. U. Straube, G. Sorge and N. R. Ivanov, Phys. Status Sol. (a), 73,467 (1982).

21. M. Sugo, M. Kasahara, M. Tokunaga and I. Tatsuzaki, J. Phys. Soc. Jpn., 53, No. 9 (1984).

*On leave from Tohoku University, Sendai 980, Japan. 